\title{
Electron Microscopic Demonstration of Centrifugal Nerve Fibers in the Human Optic Nerve
}

\section{J. Reimer Wolter}

Department of Ophthalmology, University of Michigan Medical Center, Ann Arbor, Michigan 48109, USA

Summary. Electron microscopic views of centrifugal nerve fibers in the optic nerve stump of a 56-year-old man are presented. These nerve fibers had survived for 16 days after removal of the corresponding eyeball and exhibited terminal swellings pointing in a distal direction and indicating axoplasmic flow towards the removed eye. The centrifugal nerves in this adult lack any evidence of attempted regeneration that has earlier been observed under similar conditions in the optic nerve stump of a child.

Zusammenfassung. Zentrifugale (antidrome, efferente) Nervenfasern sind hier zum ersten Mal mit dem Elektronenmikroskop im menschlichen Sehnerven dargestellt worden. Diese Nervenfasern wurden in dem Sehnervenstumpf eines 56jährigen Mannes 16 Tage nach der Entfernung des dazugehörigen Auges gefunden. Endschwellungen dieser Nervenfasern waren distal ausgerichtet und deuteten damit einen Axoplasmafluß in Richtung des entfernten Auges an. Während deutliche Regenerationsversuche an den distalen Enden unterbrochener zentrifugaler Nervenfasern im Sehnervenstumpf eines Kindes früher beobachtet worden sind, fanden sich im Sehnerven dieses Erwachsenen keinerlei Zeichen von Regeneration der zentrifugalen Fasern.

\section{Introduction}

The question of the possible existence of centrifugal (antidromic, efferent) nerve fibers in the retina and optic nerve has been of great interest to neuroanatomists as well as to ophthalmic anatomists, pathologists and physiologists for almost one hundred years. In 1957 Polyak [1] summarized the early knowledge of the subject matter by stating:

Whether all axis cylinders of the optic nerve, in man and other primates, belong to the category of afferent nerves - that is those conveying impulses generated in the retina to the brain - or whether there 
are efferent axons, or those originating in the brain and terminating in the retina, by means of which central influences may reach the photoreceptors, is a problem still to be solved. From the investigations of Ramon y Cajal, Bouin, Dogiel, Weber, and others, the presence of such fibers is fairly certain in birds and axolotl, but they have not yet been definitely shown to exist in mammals.

In 1956 the observation of numerous surviving axons in two human optic nerve stumps 12 and 16 years after enucleation was considered the first definite evidence for the existence of centrifugal nerves in the human optic nerve [2]. Terminal branches of centrifugal nerves were discovered in the normal human optic nerve and retina $[3,4]$ and it was concluded that at least some of these fibers supply blood vessels. The observation of interrupted neurites with terminal swellings pointing away from the disk in the nerve fiber layer of the diseased human retina was taken as additional evidence for the existence of centrifugal fibers [5]. Attempts at regeneration of interrupted centrifugal nerve fibers were found in a child's optic nerve stump 11 days after enucleation of the corresponding eye [6] and similar fibers seen 4 days after enucleation in the optic nerve stump of another child showed terminal swellings, but they were without any signs of regeneration [7].

In 1966 the stumps of centrifugal nerve fibers were observed in the optic nerve head of an eye that had suffered occlusion of the central retinal artery [8]. Survival of numerous nerve fibers in the optic nerves and chiasm 50 years after bilateral enucleation was recorded in 1965 as further morphologic evidence for the existence of centrifugal nerve fibers in man [9]. Photocoagulation burns in the human retina were used histologically to show reactive terminal swellings of interrupted centrifugal fibers at the proximal aspects of these burns $[10,12)$.

Sacks and Lindenberg [12] in 1969 found centrifugal nerve fibers in the optic nerve of a patient with a bilateral congenital ocular anomaly precluding the formation of retinas and estimated the number of these fibers at about $9-10 \%$ of the normal optic nerve complement. They suspected that these centrifugal fibers in their case came from the anterior hypothalamus. The exciting studies of Honrubia and Elliott $[13,14]$ have added much to the understanding of the course and the distribution of centrifugal nerves in the retina of man and monkey. These authors observed the centrifugal fibers in flat mounts of the retina to emerge from the optic disk and to form many bifurcations towards the retinal periphery. The existence of centrifugal nerve fibers in the human retina was also reported in studies by Matsuyama in 1967 [15] and by Ventura and Mathieu in 1959 and 1960 [16, 17].

Along with the morphologic findings supporting the existence of centrifugal fibers in the anterior human visual system there were numerous reports of electrophysiologic studies indicating the presence of centrifugal elements $[18,20]$. These are best summarized in Vol. 1 of [19].

All this evidence has not been sufficient, however, for complete acceptance of the presence of centrifugal fibers in the human optic nerve and retina in the literature. Brindley, in his recent book [20], for example, states in his discussion of the anatomical evidence for centrifugal fibers in the human optic nerve and retina that: "None of these constitutes compelling evidence in favour of centrifugal fibres, and there is some evidence against." Talking about the function of centrifugal fibers to the human retina, Brindley says: "If I take a more sceptical attitude towards the question of centrifugal fibers it is in part because of the difficulty of finding any convincing suggestions of how the centrifugal control of retinal activity postulated 


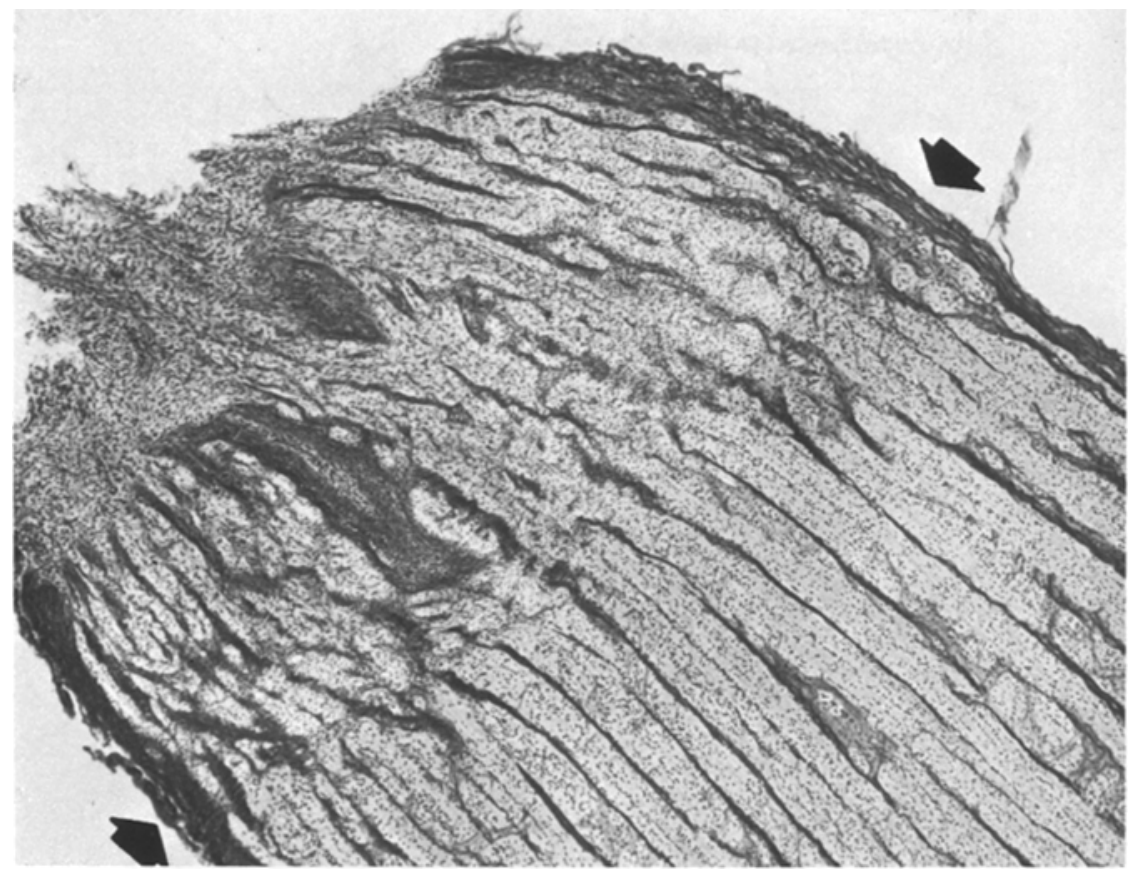

Fig. 1. Longitudinal section of the optic nerve stump in the present case with its distal end pointing to the left and up. The zone of total destruction caused by the surgical cut 16 days before the removal of this nerve is indicated by two arrows. Formalin fixation, frozen section, pyridine silver stain, photomicrograph $\times 100$

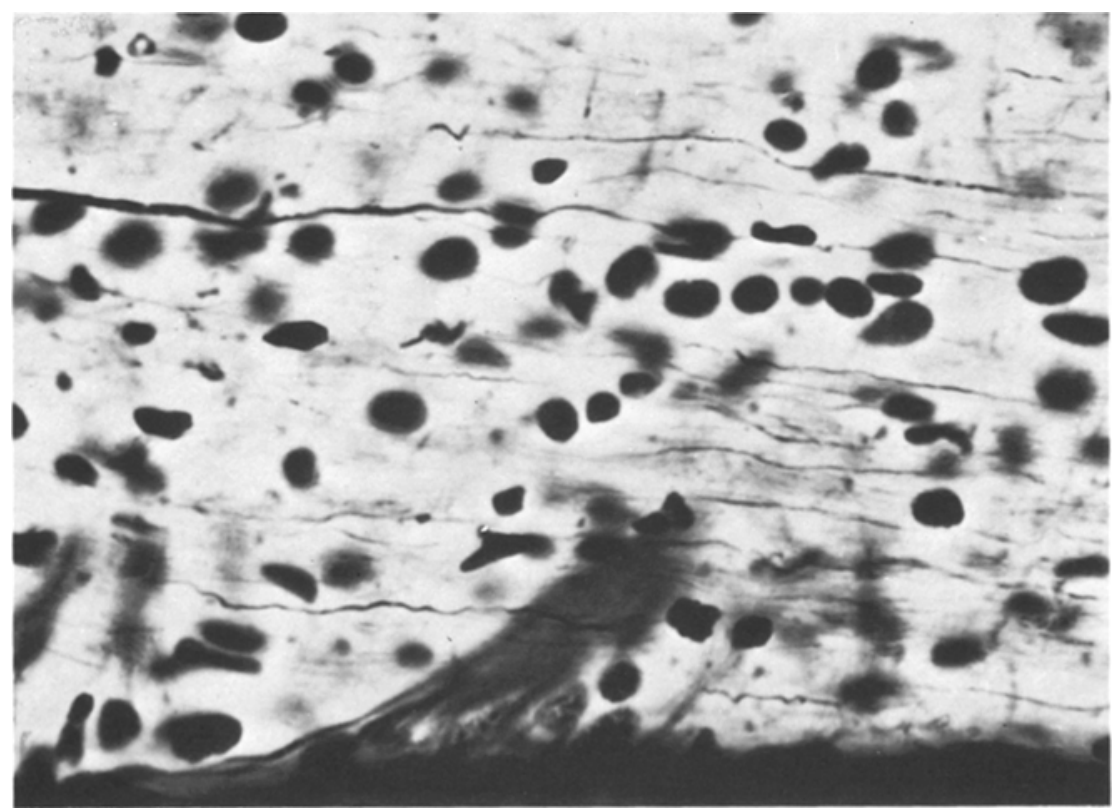

Fig. 2. Reduced number of nerve fibers found in the optic nerve stump of the present patient running from left to right in the picture. The nuclei of neuroglia are also stained. Longitudinal frozen section, pyridine silver stain, photomicrograph $\times 640$ 
J.R. Wolter

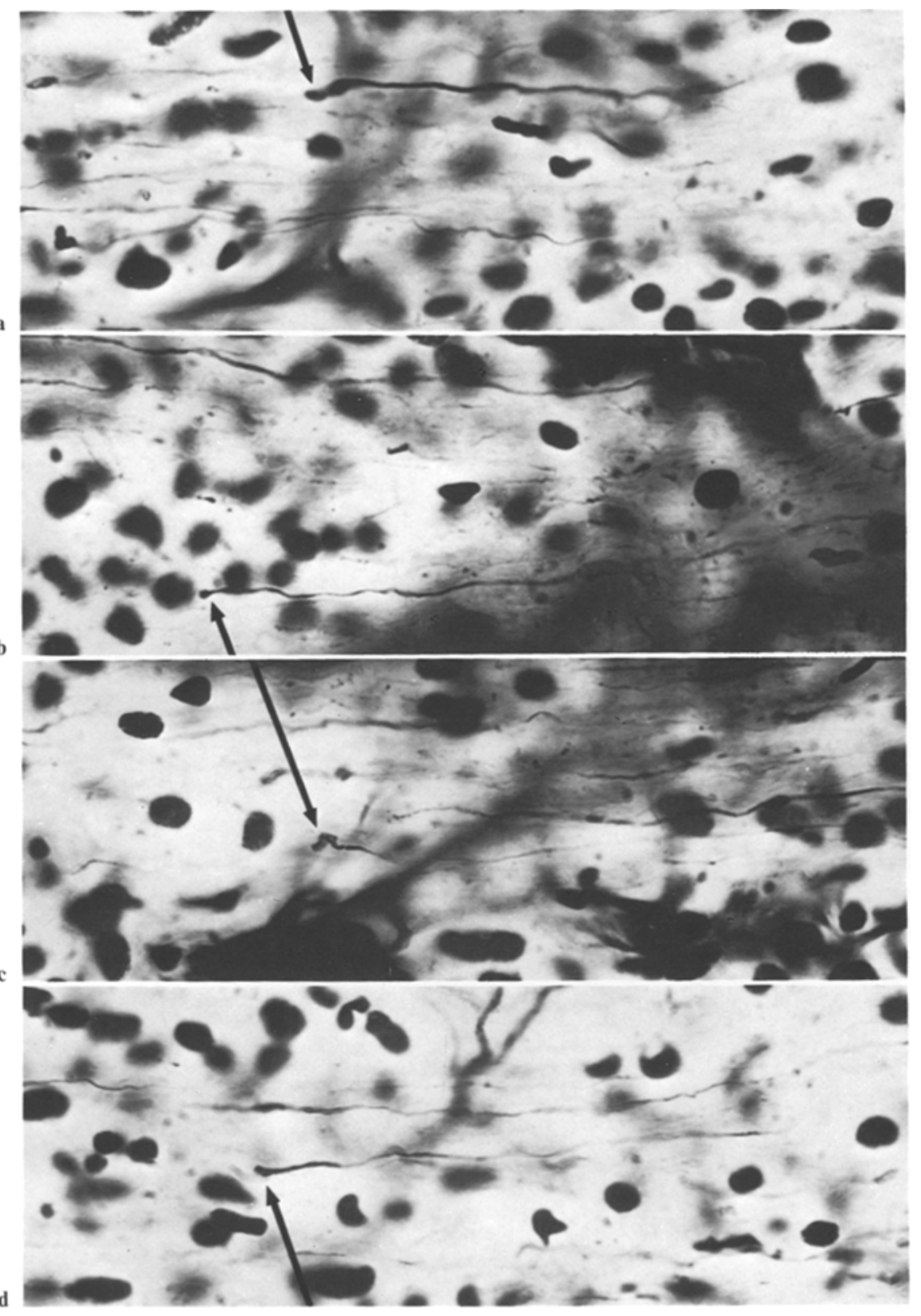

Fig. 3. Four photographs (a, b, $\mathbf{c}$ and $\mathbf{d}$ ) showing club-like terminal swellings of centrifugal nerves (arrows) pointing towards the distal end of the optic nerve stump in the present case. Longitudinal frozen sections, pyridine silver stains and photomicrographs $\times 640$ 


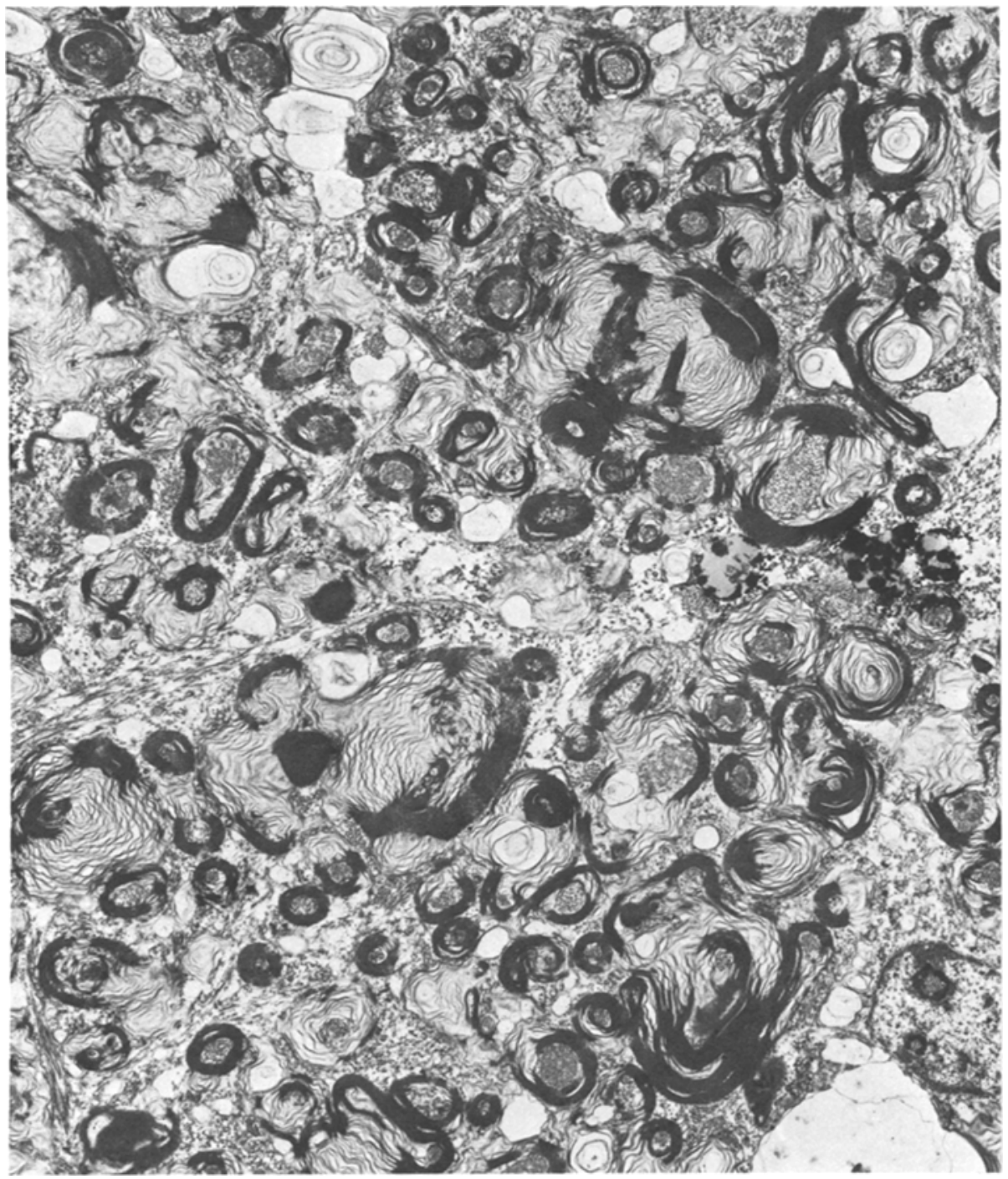

Fig. 4. Cross section of the optic nerve stump in the present case seen with the electron microscope. Many artifacts are observed due to formalin fixation, but numerous surviving axons are clearly visible in spite of these. Formalin fixation, cross section, magnification $\times 4,800$

could be of sufficient use - to justify the occupation of so much valuable space in the overcrowded optic nerve...". Statements like this are surprising to all those who have seen the anatomic and physiologic evidence for the existence of these centrifugal fibers.

It is the purpose of the present paper to give convincing additional evidence for the presence of centrifugal nerve in the human optic nerve. 


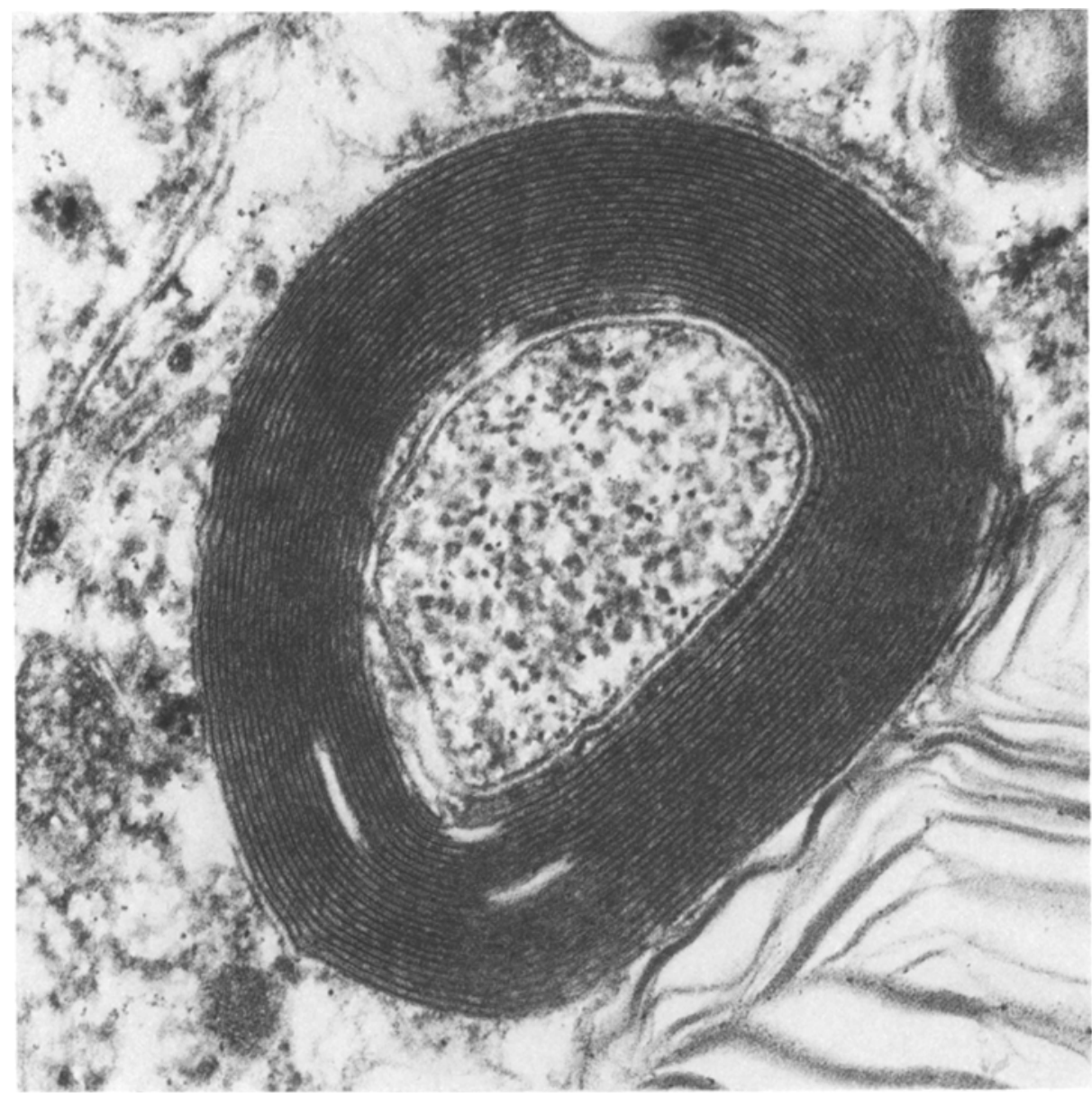

Fig. 5. High power view of one well-preserved axon with its distinct myelin sheath seen in a cross section of the present optic nerve stump. Formalin fixation, electron micrograph $\times 80,000$

\section{Case Report}

This 56--year-old white male had noted a shadow coming over his left visual field early in May 1977. His local ophthalmologist made a clinical diagnosis of malignant melanoma in the left eye. This eye was enucleated on 15 June 1977 and the eye was sent to me for histopathological study.

The eye was of normal size. External examination revealed a dark brown tumor on the outside of the sclera in the area of the inferior nasal vortex vein. Transillumination showed the shadow of a large intraocular tumor involving the inferior nasal portion of the choroid. After opening the eye in the plane of the extraocular tumor extension the intraocular tumor was also found to be of brown color and it measured $15 \times 15 \times 10 \mathrm{~mm}$. Histologic sections showed the anterior segment of this eye to be normal, except for some diffuse mononuclear infiltration in the iris and ciliary body. A serous transudate was seen under the retina on and next to the tumor. The outer retinal layers and the pigment epithelium exhibited degenerative changes in the region of the tumor. Bruch's membrane had remained intact, however, and many drusen were observed on its surface in the area of the choroidal tumor. The choroidal tumor was composed of rather irregular cells most of which were unpigmented. Some of the 


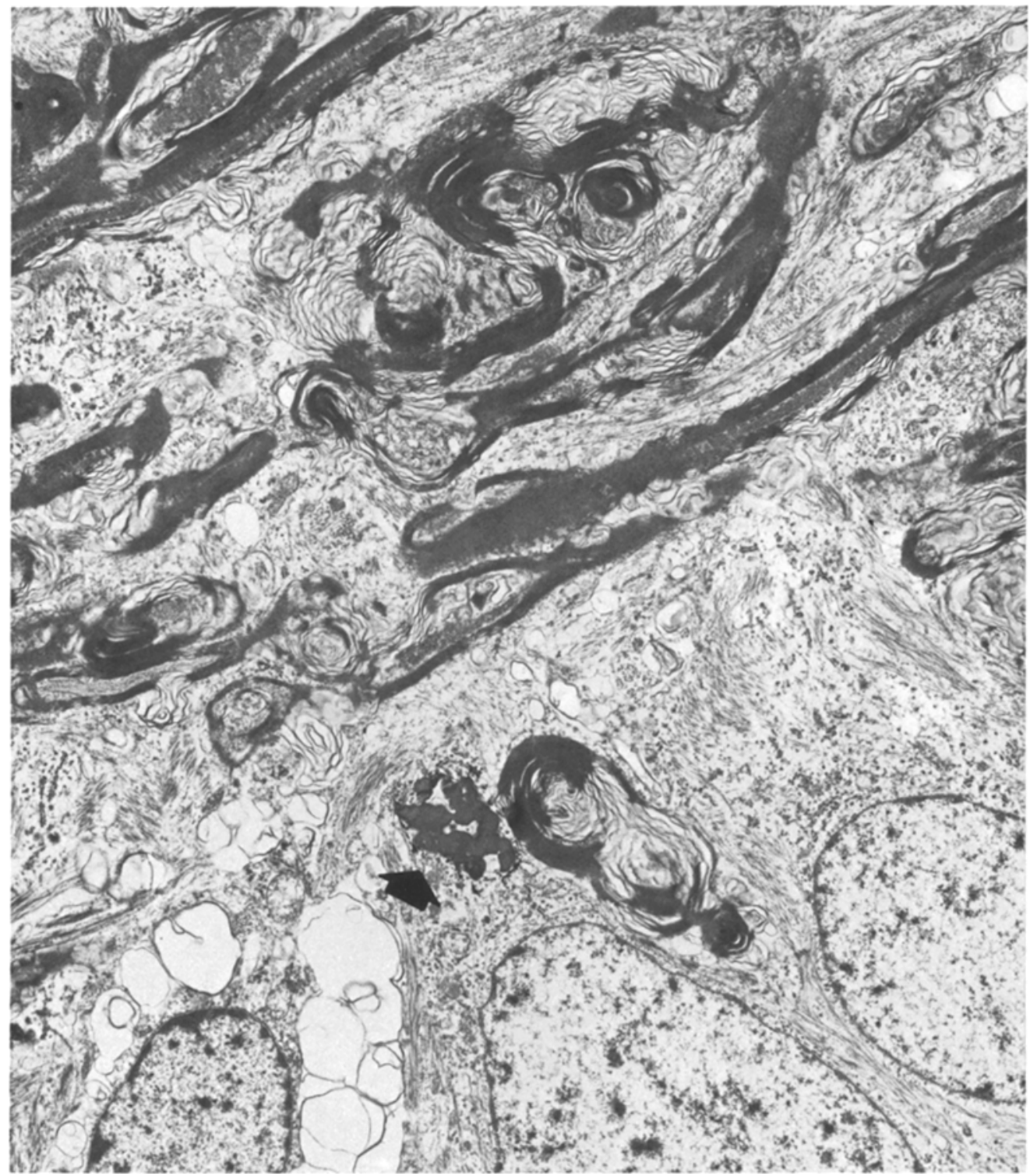

Fig. 6. Electron micrograph of longitudinal section from an area near the distal end of the present optic nerve stump showing several terminal swellings (upper half of picture), a piece of myelin (arrow) and nuclei of active glia (lower half of picture). Formalin fixation, electron micrograph $\times 15,000$

cells were spindle-shaped whereas others were of an epithelioid cell type. Most of these cells had nucleolated nuclei and occasional tumor giant cells were recognized. On its scleral side, the tumor was seen to extend through the sclera along the pathway of the vortex vein. On the outside of the sclera the tumor formed a relatively well-defined nodule that appeared to have been removed as a whole. The optic nerve was not involved by the choroidal neoplasm and appeared normal. A diagnosis of a large malignant choroidal melanoma of a mixed spindle B and epithelioid type with direct extraocular extension was made. Additional surgical removal of orbital contents was recommended. 


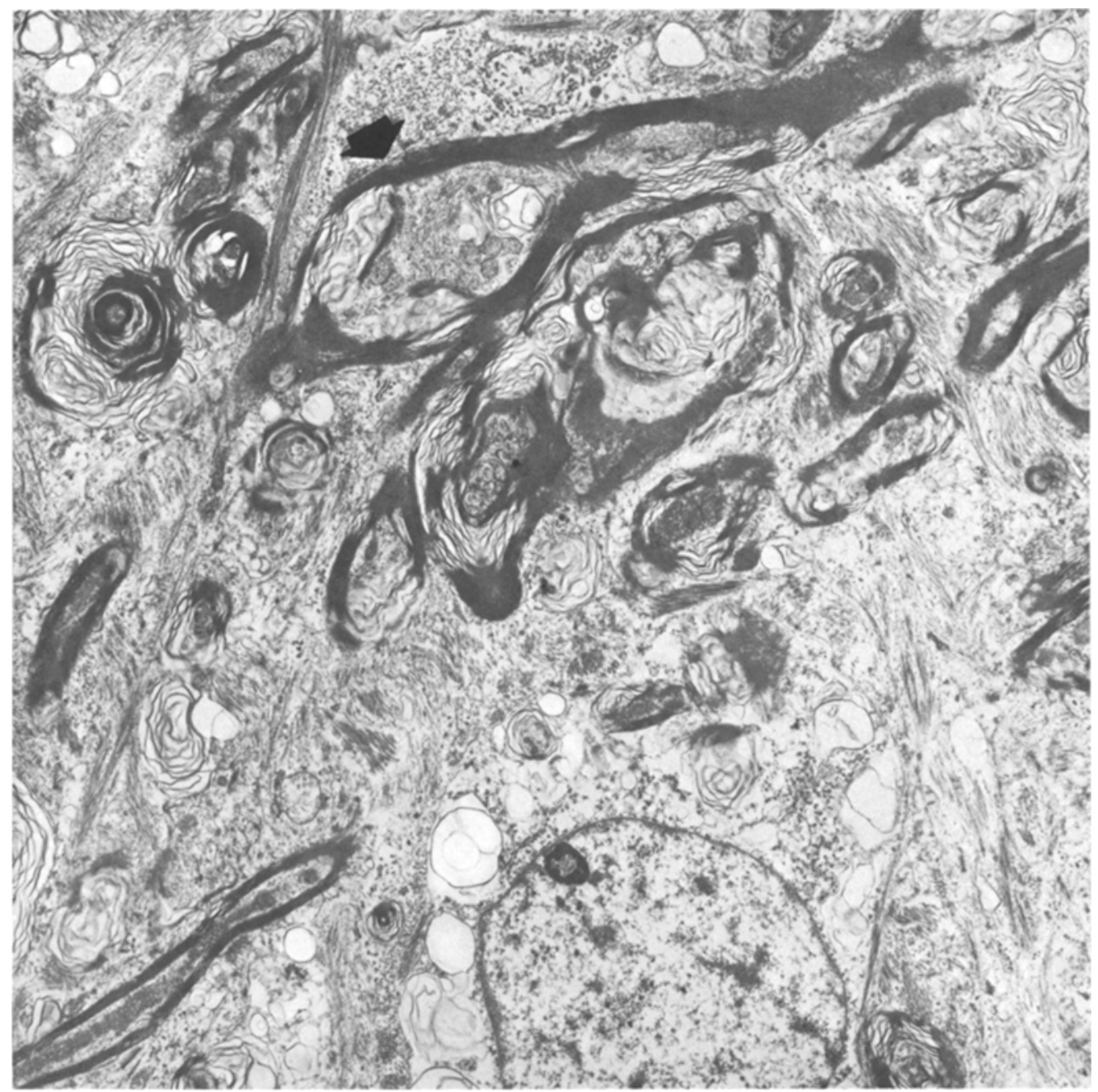

Fig. 7. Terminal swelling of a centrifugal nerve (arrow) seen in longitudinal section. Nucleus of a glial cell and a well-preserved axon seen in the lower part of the picture. Formalin fixation, electron micrograph $\times 15,000$

The patient was admitted to this University Hospital on 30 June 1977. A partial exenteration of the left orbit was done under general anesthesia on 1 July 77 without difficulties. Along with the inner portions of both lids, the conjunctival sac and the structures of the muscle cone of the left orbit containing a mesh-covered plastic implant as well as the optic nerve stump measuring $8 \mathrm{~mm}$ in length, were obtained. Histological study of these tissues did not reveal additional malignant melanoma.

The optic nerve stump was isolated after proper fixation in formalin and cut lengthwise on the freezing microtome. The pyridine silver carbonate stain of Hicks [21] was used. Next to the distal end of the optic nerve stump there was a sharply limited zone of total destruction of all nerve fibers and most of the glia (Fig. 1). Active microglia was accumulated in this zone and filled the space of the absent neuroectodermal elements. The number of axons in this optic nerve stump was found greatly reduced as compared to the number of axons seen in the normal human optic nerve. However, numerous axons 
were present and their number was estimated to be about one-tenth of that found in a normal optic nerve (Fig. 2).

Next to the zone of destruction at the end of the optic nerve stump there were numerous axons pointing with terminal swellings (end bulbs) towards the distal cut end of the nerve stump. These terminal swellings were mostly simple club-like formations with more complex structures in a few instances. No evidence of attempted regeneration was recognized (Fig. 3).

Pieces of optic nerve tissue from the zone where most of the terminal swellings had been observed by silver stain were isolated for electron microscopy. Electron micrographs clearly show the surviving nerve fibers to be surrounded by dark-staining myelin (Figs. 4 and 5). In many instances the electron micrographs also show portions of the swollen distal ends of the nerve stumps (Figs. 6 and 7). These terminal swellings are also almost completely covered by the extended myelin sheath, but defects are seen through which the swollen substance of the axon appears to be exposed. Active glia is demonstrated between the nerve fibers (Figs. 4-7). Very few irregular pieces of myelin (Fig. 6) are all that is left of the great number of afferent (centripetal) nerve fibers which have undergone degeneration after the removal of the eye.

\section{Discussion}

The observation of living nerve fibers in the optic nerve stump of the present patient 16 days after removal of the corresponding eye is in itself believed to be new evidence for the existence of centrifugal (antidromic, efferent) nerve fibers in the anterior human visual system. All afferent nerve fibers are expected to have degenerated and disappeared within a few days after the removal of the eye. All afferent nerve are axonal processes of ganglion cells located in the retinal nerve fiber layer of the removed eye, of course.

Interrupted centrifugal nerve fibers in the optic nerve can be recognized by the direction of their terminal swellings. These point in a distal direction, and most of them are found on the distal end of the optic nerve stump next to the separating surgical cut. These terminal swellings indicate eaxoplasmic flow towards the distal end of the optic nerve stump.

Early attempts at regeneration starting out from the terminal swellings of interrupted centrifugal nerves were observed in earlier studies [6] in the optic nerve stump of a child 11 days after enucleation of the corresponding eye, but this change was not seen 4 days after enucleation in another child [7]. The absence of any attempts at regeneration in the present case of a56-year-old man studied 16 days after enucleation is of interest. It may indicate that the regeneration potential of the centrifugal fibers may decrease with advancing age. This fits the basic concept that repair, regrowth, and regeneration in general are most exuberant early in life and decrease with age.

The number of surviving nerve fibers in the present optic nerve stump was again estimated at about $10 \%$ of the number of fibers seen in a normal human optic nerve. This observation is in good agreement with our own earlier estimates [9] and with that of Sacks and Lindenberg [12].

The present paper offers the first electron microscope view of centrifugal fibers of the human optic nerve. It is obvious that the numerous well-preserved axons with their myelin sheaths found in the optic nerve stump cannot be afferent nerves originating in the retina of the corresponding eye that was removed 16 days before. These axons were alive and connected to their ganglion cells somewhere in the brain 
at the time of the removal of the nerve stump. The electron microscopic demonstration of these nerves is convincing evidence for the presence of centrifugal (efferent) fibers in the human optic nerve. Electron microscopy was used not only to confirm the presence of nerve fibers in this optic nerve stump, but also to get a view of the terminal swellings. It was no surprise that the centrifugal nerve fibers are myelinated and resemble in the electron micrograph by size and shape all other nerve fibers seen in the human optic nerve under normal conditions [20,22]. It is of some interest that the myelin sheath is seen to extend in an attempt to surround the terminal swellings. However, there appear to be defects in this covering where the swollen substance of the axonal core is exposed.

The observation of efferent nerve fibers in the human inner ear are an interesting parallel to the findings in the optic nerve discussed in this paper [23-25]. These nerves are sometimes called the olivo-cochlear bundle and they are believed to control the auditory input at the periphery. Their function has been compared to a feedback loop and modern neurophysiologists and neuroanatomists believe that the brain in all instances controls the sources of its information by sending out centrifugal (efferent) nerve fibers in the peripheral receptors.

The present study again is only a very small step towards a better understanding of the efferent component of the anterior visual pathway in man. However, it is hoped that it will stimulate the interest of those investigators who have the knowledge and the facilities to reveal the origin and function of this efferent nerve system.

\section{References}

1. Polyak, S.L.: The Vertebrate Visual System, p. 292. Chicago: University of Chicago Press 1957

2. Wolter, J.R., Liss, L.: Zentrifugale (antidrome) Nervenfasern im menschlichen Sehnerven. Albrecht v. Graefes Arch. Ophthal. 158, 1 (1956)

3. Wolter, J.R.: Über Endigungen zentrifugaler Nervenfasern an den Blutgefäßen der menschlichen Netzhaut. Albrecht v. Graefes Arch. Ophthal. 158, 524 (1957)

4. Liss, L., Wolter, J.R.: Zur Innervation der Blutgefäße des menschlichen Sehnerven. Klin. Monatsbl. Augenheilkd. 129, 793 (1956)

5. Wolter, J.R.: Ein weiterer Beweis für die Existenz zentrifugaler Nervenfasern in der menschlichen Netzhaut. Albrecht v. Graefes Arch. Ophthal. 158, 235 (1956)

6. Wolter, J.R.: Regenerative potentialities of the centrifugal fibers of the human optic nerve. A.M.A. Arch. Ophthalmol. 64, 697 (1960)

7. Pfister, R.R., Wolter, J.R.: Centrifugal fibers of the human optic nerve. Neurology (Minneap.) 13, 38 (1963)

8. Wolter, J.R.: Centrifugal nerve at the optic disk (ten days agter complete occlusion of the retinal artery). Excerpta Med. Int. Congr. Ser. 146, 117 (1966)

9. Wolter, J.R.: The centrifugal nerves in the human optic tract, chiasm, optic nerve and retina. Trans. Am. Ophthalmol. Soc. 63, 678 (1965)

10. Wolter, J.R., Moorman, L.T.: Early effects of photocoagulation on the nerve fiber layer of the human retina. Arch. Ophthalmol. 76, 385 (1966)

11. Wolter, J.R., Lund, O.E.: Reaction of centrifugal nerves in the human retina two weeks after photocoagulation. Trans. Am. Ophthalmol. Soc. 66, 173 (1968)

12. Sacks, J.G., Lindenberg, R.: Efferent nerve fibers in the anterior visual pathway in bilateral congenital cystic eyeballs. Am. J. Ophthalmol. 68, 691 (1969)

13. Honrubia, F.M., Elliott, J.H.: Efferent innervation of the retina. I. Morphologic study of the human retina. Arch. Ophthalmol. 80, 98 (1968) 
14. Honrubia, F.M., Elliott, J.H.: Efferent innervation of the retina, II morphologic study of the monkey retina. Invest. Ophthalmol. 9, 971 (1970)

15. Matsuyama, M.: Existence of centrifugal nerve fiber in the retina. Jahresbericht Kurashiki Zentral hospital, 36, 1 (1967)

16. Ventura, J., Mathieu, M.: Exogenous fibers of the retina. Trans. Can. Ophthalmol. Soc. 22, 184 (1959)

17. Ventura, J., Mathieur, M.: Silver impregnation of whole retinas. A.M.A. Arch. Ophthalmol. 64, 528 (1960)

18. Dodt, E.: Centrifugal impulses in rabbit's retina. J. Neurophysiol. 19, 301 (1956)

19. Walsh, F.B., Hoyt, F.H.: Clinical Neuro-Ophthalmology. Baltimore: Williams and Wilkins 1969

20. Brindley, G.S.: Physiology of the Retina and Visual Pathway, pp. 106 and 109. Baltimore: Williams and Wilkins 1970

21. Hicks, S.P.: A rapid pyridine silver stain for nervous tissue and reticular fibers. J. Lab. Clin. Med. 31, 2 (1977)

22. Fine, B.S., Yanoff, M.: Ocular Histology, p. 243. New York: Harper and Row 1972

23. Ross, M.D.: The general visceral efferent component of the eighth cranial nerve. J. Comp. Neurol. 135, 453 (1969)

24. Ross, M.D.: Fluorescence and electron microscopic observations of the general visceral, efferent innervation of the inner ear. Acta Oto-Laryngol. [Suppl.] (Stockh.) 286, 1971

25. Ross, M.D.: Autonomic Components of the VIIIth Nerve. Adv. Otorhinolaryngol. 20, p. 316-336 (1973)

Received August 11, 1978 